\title{
Menonitas y deforestación en América del Sur
}

\author{
Mennonites and deforestation in South America
}

Marc Dourojeanni

Universidad Nacional Agraria de La Molina. Lima, Perú

Recibido: 27/10/2021 Revisado: 18/12/2021_Aceptado: 23/12/2021Ｐublicado: 15/01/2022

\section{RESUMEN}

Los menonitas han llegado a América del Sur en números considerables después de la Segunda Guerra Mundial y se han instalado en varios países, pero principalmente en Paraguay y Bolivia donde desarrollaron grandes emprendimientos agropecuarios, ocupando bosques y otros ecosistemas naturales. Se estima que ya han deforestado más de cuatro millones de hectáreas de los biomas Chaco y Chiquitanía, principalmente para ganadería y soya. A partir del presente siglo comenzaron a invadir la Amazonia de Bolivia y más recientemente las de Perú y Colombia, habiendo ya deforestado posiblemente unas 20 mil hectáreas. Por sus antecedentes en México y América Central, así como en Paraguay y Bolivia, también preocupa el hecho de que sus tácticas para ocupar la tierra, tanto como muchas de sus prácticas, serían en gran medida ilegales y perjudiciales tanto al ambiente como a las poblaciones locales, especialmente indígenas. Evidencias recientes de Perú y Colombia confirman esas sospechas. Palabras clave: Amazonia, menonitas, deforestación, ocupación ilegal de la tierra, impacto ambiental y social

\begin{abstract}
Mennonites migrated to South America in considerable numbers after World War II and have settled in several countries, but mainly in Paraguay and Bolivia where they developed large agricultural enterprises, occupying forests and other natural ecosystems. It is estimated that they deforested over four million hectares of the Chaco and Chiquitania biomes, mainly for livestock and soybean. Since the current century they began to invade the Amazon of Bolivia and more recently those of Peru and Colombia, having already deforested probably around twenty thousand hectares. Given its antecedents in Mexico and Central America, as well as in Paraguay and Bolivia, there is also concern that its tactics to occupy the land, as well as many of its practices, may be largely illegal and harmful to both the environment and local population, especially indigenous people. Recent evidence from Peru and Colombia confirms these suspicions.
\end{abstract} Keywords: Amazonia, Mennonites, deforestation, unlawful land occupation, environmental and social impacts. 


\section{INTRODUCCIÓN}

Los menonitas son una secta religiosa que pregona y practica una interpretación de la Biblia que se traduce en una vida simple y muy apegada a la tierra (Casell 2016). Ellos, por sus creencias, son pacíficos y discretos y nada de lo que ellos creen parece perjudicar a los demás. El problema es que, aunque vivan simplemente, sin acumulación de riqueza ni poses superfluas, ellos son agricultores muy eficientes que usan tecnología convencional moderna. Por eso, dependiendo de donde se instalan, sus actividades pueden ser altamente impactantes en el ambiente. Como suelen asentarse en tierras cubiertas de bosques y vegetación naturales su primera acción es eliminar los árboles, aprovechar la madera y transformar el lugar en un paisaje típicamente agropecuario. Eso, que hasta medio siglo atrás, era por muchos considerado un hecho positivo, es ahora un problema crecientemente serio ya que, en toda América del Sur, la deforestación y la contaminación ambiental, así como sus consecuencias, han superado el límite de lo tolerable para el bienestar de la humanidad (De Syl et al 2015; Ritchie y Roser 2021).

El tema de los asentamientos menonitas es relativamente nuevo en el Perú y en Colombia, pero es bien conocido en varios otros países y biomas de América Latina, y es especialmente crítico en México, Paraguay, Bolivia y Centroamérica (Fast 2018, Griesbrecht y Klausen 2015, Giesbrecht 2020, Le Polais de Waroux et al 2020), por los considerables los impactos ambientales que ocasionan y por la relativa impunidad con que estos se cometen. En este artículo se revisan esos problemas y se detalla lo que está ocurriendo en América del Sur, especialmente en la Amazonía peruana donde los menonitas operan desde 2016, caso en el que en dos locali- dades ya deforestaron quizá más de cuatro mil hectáreas de bosque natural en abierta violación de la legislación vigente (Praeli 2020b, Dourojeanni 2020, Praeli 2021a; 2021b).

\section{Menonitas en América Latina}

La historia de los menonitas comenzó en 1523 en la Suiza germánica, cuando un sacerdote católico inició una reforma que se dispersó y consolidó rápidamente en el Sacro Imperio Germánico. A partir de 1529 esos disidentes pasaron a ser perseguidos y martirizados. En 1536 el sacerdote católico Menno Simons, influyente contestatario, también rompió con la Iglesia Católica y se unió a ellos, por entonces más conocidos como anabaptistas, por la forma poco ortodoxa en que adoptaron ese sacramento. Menno, a pesar de la persecución, tuvo éxito en divulgar las nuevas creencias las que se propagaron especialmente a Holanda y Polonia, y luego a otros países del este europeo, pasando a ser conocidos como menonitas. En 1683 se produjo la primera emigración menonita a Pennsylvania, que era parte de las colonias inglesas en América. Esas migraciones continuaron durante el siglo XVIII. La vida de los que se quedaron en Europa era cada vez más dura, especialmente en Prusia, de tal modo que, en 1788, por invitación de la emperatriz Catalina la Grande, ellos emigraron a Rusia con varias ventajas, entre ellas la posesión de tierras. Con los cambios políticos en Rusia esos beneficios fueron mermados por lo que se intensificó la migración a Norte América, inclusive al Canadá (1873). La primera colonia agrícola menonita en América Latina se desarrolló en Argentina, en 1877. En términos de prácticas religiosas y en función de sus orígenes los menonitas se han subdividido en diversos grupos, pero mantienen bastante cohesión (Dyck1993; Casell 2016). 
En el Siglo XX, la revolución bolchevique y las matanzas estalinistas obligaron a muchos menonitas a huir de Rusia. Entre 1920 y 1930 parte de ellos fueron a Canadá, y también a México (1922) y Paraguay (1930). La Segunda Guerra Mundial trajo más persecuciones para ellos, por lo que se produjeron nuevas migraciones a EE.UU., Canadá, Belice y México. En 1945 llegó otra ola de refugiados, que se instalaron en Uruguay, Brasil y Paraguay. Muchos países latinoamericanos invitaron, facilitaron y hasta financiaron (México, por ejemplo) la venida de contingentes de menonitas a desbravar tierras "vírgenes" o "desocupadas sin uso", es decir, espacios naturales, en general bosques, que les fueron dados en propiedad, entre otros privilegios, muchas veces previamente decididos mediante convenios. La idea detrás de esa generosidad fue, en general, ocupar territorios considerados subutilizados o supuestamente codiciados por otros países, promover el crecimiento económico, mostrar a la población local el ejemplo de un "buen uso de la tierra" (Goosen 2016) y, sin duda, también estaba latente el objetivo de "mejorar la raza" trayendo personas blancas, idea que era tan común en el Perú (Maguiña 2010; Gallirgos 2015) como en otros países de la región hasta mediados del siglo pasado. En las últimas décadas se han acelerado los movimientos migratorios menonitas, a veces entre países de la región, en función de los cambios políticos y de la aparente disponibilidad de tierras, siendo evidente un movimiento muy reciente hacía Perú (Servindi 2019, Praeli 2020, 2021a, 2021b, Dourojeanni 2020) y Colombia (Betancourt 2018).

En 2015, según fuentes menonitas (GAMEO, 2015), existían 200 mil menonitas asentados en América Latina, siendo las colonias más importantes las de México (34 mil) y América Central (50 mil), seguidas de las de Paraguay (35 mil), Bolivia (27 mil) y Brasil (15 mil). Pero, hay menonitas que viven en zonas urbanas de todos los países. De otra parte, muchas fuentes creíbles citan números mucho más expresivos de menonitas para América Latina, totalizando desde 270 mil (Wikipedia 2021a) hasta cerca de 700 mil personas (Wikipedia 2021b). De hecho, buscando información país por país, los números se acercan a las figuras más abultada $^{1}$. Por ejemplo, se cita de 90 mil a 100 mil menonitas apenas en México (Quadri 2017; Morimoto 2019). En Paraguay hay más de 67 mil menonitas (Correia 2020) y casi 60 mil en Bolivia en 2015 (Kopp 2015). Y, como dicho, hay menonitas en todos los países de la región.

\section{Deforestación}

El principal, más notorio y denunciado impacto ambiental de la actividad de los menonitas en América Latina ha sido y sigue siendo la deforestación y la destrucción de otros ecosistemas naturales, muy frecuentemente sin autorización. Para discutir ese tema es importante separar dos situaciones. En efecto, hasta fines de la década de 1980, la sociedad no consideraba que la expansión de la frontera agropecuaria sobre los bosques naturales era un problema ambiental. Eso era preocupación de expertos (Dorst, 1965; Goodland e Irwin 1975) y muy pocos eran los gobiernos de la región que ya tenían o anunciaban políticas para controlarla. Al contrario, en especial en el lapso (1920-1960), en que los menonitas llegaron en gran número a América Latina, deforestar era considerado una "mejoría" deseable. Por eso, aunque a continuación se menciona hasta dónde fue posible las extensiones de tierra

\footnotetext{
${ }^{1}$ Parece que la información oficial menonita sobre su población en los países en que habitan (GAMEO y otras fuentes de ellos) es, por algún motivo, sistemáticamente muy inferior a la que proveen los gobiernos y los estudiosos.
} 
ocupadas por ellos, pues eso revela su impacto en los biomas de la región, es más importante analizar las deforestaciones recientes, en especial en el Siglo XXI, cuando nadie, ni los menonitas, podían ignorar las consecuencias de practicar deforestación en gran escala.

A continuación, se pasa revista a lo que se sabe de la situación de los asentamientos menonitas en Bolivia, Paraguay y Perú, donde afectaron mucho los biomas Chaco y Chiquitanía y, más recientemente, el Amazónico, proceso que también está comenzando en Colombia. Hay menonitas en el Brasil, donde llegaron entre 1928 y 1934 y se instalaron en las montañas de Santa Catarina. Pero no se adaptaron y se trasladaron cerca de la ciudad de Curitiba, en Paraná, no habiéndose dispersado más (Luz et al 2014). En el caso de Ecuador, similarmente, existen menonitas hace tiempo (Moya 2020) pero no hay registro de que se dediquen a actividades agropecuarias. No se ha conseguido, para todos los países, información diferenciada y consolidada sobre la extensión ocupada por las propiedades menonitas en cada país, pero no cabe duda de que puede ser muy grande.

El primero de los países citados en recibir menonitas fue Paraguay. El Gobierno paraguayo, como otros, aceptó los menonitas teniendo en mente disuadir pretensiones territoriales de sus vecinos y, por eso, les brindó facilidades. Comenzaron a llegar en la década de 1920 (Goossen 2016). En 1927, se instalaron en el Chaco, fundando la grande y famosa colonia Filadelfia. La mayoría de los 19 asentamientos menonitas del país están en el Chaco y albergarían actualmente a unas 67 mil personas (Correia, 2020) pero los hay también en la región oriental del país. Cuando llegaron al Chaco, ese territorio era salvaje, estando solo ocupado por indígenas no contactados o en estado inicial de contacto. El desarrollo, lento y difícil al comienzo, fue facilitado por los avances de la carretera Trans Chaco (Hecht 2007) y pasó a ser muy rápido y, así, en 2011 los tres primeros asentamientos chaqueños ya faenaban 360 mil cabezas de ganado vacuno por año, habiendo transformado el Paraguay en el sexto productor mundial de carne (Duerksen 2011). Es probable que entonces sus pastizales ya debían cubrir varios cientos de miles de hectáreas y, además, usaban de 25 a 30 mil hectáreas por año para cultivos agrícolas. Asimismo, crearon una cooperativa indígena que tenía 123 mil hectáreas, de las que 27 mil eran pastizales. Pero esas cifras corresponden tan solo a una parte de sus posesiones en el país pues hubo menonitas que se separaron de las colonias. En esa región, una sola familia menonita podía disponer de 2 a 3000 hectáreas, por las que oportunamente pagaron muy poco por ser "tierras sin desbravar" (Daniels 2011). Vidal (2010) reporta que tan solo en el Chaco los menonitas ya debían poseer tanto como dos millones de hectáreas, a las que deforestaban con urgencia, precisamente debido al riesgo de que las crecientes protestas de los ambientalistas y antropólogos consiguieran imponer limitaciones. Además, comenzaron a sufrir presión de otros usuarios de la tierra (Mander 2019). El reciente estudio de le Polain de Waroux (2020) confirma que las colonias menonitas controlan hoy alrededor de $1,8 \mathrm{mi}$ llones de hectáreas en Paraguay, es decir 4,5\% del territorio nacional. A esto hay que sumar los cientos de miles de hectáreas de tierra de propiedad privada de menonitas fuera de las colonias, que en 2010 llevaba este total casi al $8 \%$ del territorio del Paraguay (Fast 2020). En promedio, según deducen le Polain de Waroux (2020), los menonitas controlan unas veinte veces más tierras que los propios paraguayos, 
a pesar de ser el 0,45\% de la población del país.

Los menonitas llegaron a Bolivia a partir de 1954. Pero la mayor expansión se dio a partir de 1990, aprovechando de un programa de ampliación de la colonización de las tierras bajas (Chaco y Chiquitanía) financiado por el Banco Mundial. Unos 10 mil de ellos se instalaron cerca de la ciudad de Santa Cruz, donde adquirieron inicialmente unas 70 mil hectáreas que habían sido propiedad de ganaderos y pequeños propietarios o poseedores locales. Este núcleo se expandió y multiplicó rápidamente en la región de Santa Cruz y, en mucho menos proporción, en otras. En 2008 la población menonita alcanzó 57375 habitantes (Schartner y Dürksen 2009). A 2015 existían 67 colonias o emprendimientos menonitas en el país, cubriendo oficialmente más de 324 mil hectáreas. Pero, esta información solo se refiere a las colonias y propiedades ya tituladas y debidamente registradas en el Instituto de Reforma Agraria, existiendo muchas otras bien establecidas y reconocidas, pero no legalizadas ni contabilizadas. En total se estima que los menonitas posean unas $650 \mathrm{mil} \mathrm{hec-}$ táreas del territorio boliviano (Kopp 2015). Esas tierras eran, en su mayor parte, bosques chiquitanos y chaqueños no intervenidos, cuya madera fue explotada por los menonitas antes de quemar el resto para establecer sus crianzas y cultivos. En total, según el recuento de le Polain de Waroux (2020), los menonitas de Bolivia cultivan actualmente más de un millón de hectáreas en las tierras bajas, principalmente en el departamento de Santa Cruz en el que tienen alrededor de 875 000 ha. Ellos han aprovechado del auge del cultivo de la soya, que se ha convertido en el cultivo más importante de esa parte del país. Los menonitas no habían penetrado en el bioma amazónico de Bolivia hasta que, a partir de 2005 se han instalado en Río Negro, Beni, cerca de la frontera con el Brasil. La colonia de Río Negro, después de un periodo de poca actividad, relanzó sus actividades, causando la destrucción de 5 mil hectáreas de bosque entre 2017 y 2019 (Finer y Mamani 2019).

Los registros de menonitas dedicados a actividades rurales en Colombia son más recientes. Las primeras denuncias corresponden a la adquisición, en 2016, de 17 mil hectáreas en el departamento de Meta (El Tiempo. Unidad Investigativa 2018; Impacto 2020), en el centro del país, que ecológicamente corresponde a la Amazonía Andina, aunque se encuentra en la cuenca del Orinoco. Compraron algunas propiedades grandes y muchas pequeñas, pagando al contado. Se trata, por el momento, de unas 300 personas principalmente provenientes de México, que piensan dedicarse al cultivo de soya, entre otros. Crearon dos sociedades o empresas para ese fin: Agroindustria Llanos la Esperanza S. A. S. y Agrícola Enns del Llano S. A. S. Las tierras adquiridas parecen todas haber sido habilitadas para uso agropecuario desde hace algún tiempo. Según Rutas del Conflicto (2021) y La Liga Contra el Silencio (2021), este grupo ya ha acumulado en los Llanos no menos de 32552 hectáreas. Uno de estos predios todavía es un baldío de la nación. También describen que varios predios les fueron adjudicados fraudulentamente (Rutas del Conflicto 2021).

El caso peruano ha sido muy bien descrito por Praeli (2020a, 2020b, 2021a, Actualidad Ambiental 2021) y claramente demostrado por los estudios del Proyecto de Monitoreo de los Andes Amazónicos - MAAP (Finer y Mamani 2019; Finer et al 2020). Aunque los menonitas llegaron al Perú en los años 1950, ellos se dedicaron al proselitismo religioso y hasta 
recientemente no se tenía noticia de grupos practicando agricultura en el Perú (Wikipedia 2021c). Se conocía la presencia en la Amazonía, desde algunas décadas atrás, de otras sectas religiosas, como la Asociación Evangélica Misionera Nuevo Pacto Universal, más conocidos como los "israelitas", las que se han reactivado recientemente (Praeli 2021b) ahora reforzados por un partido político (Villasante 2020). Pero, menonitas provenientes principalmente de Bolivia han adquirido, a partir de 2015 una extensión indefinida pero grande de tierras cubiertas de bosques naturales en dos localidades de la Amazonía peruana: Masisea (Ucayali) y Tierra Blanca (Loreto), en las que hasta octubre de 2020 ya habían deforestado un total de 3400 hectáreas (Finer et al 2020). A pesar de la intervención de la Fiscalía, de la Procuraduría Pública del Ministerio del Ambiente y de la autoridad forestal regional que respondieron a demandas sobre la legalidad de la operación, ellos han continuado deforestando y abriendo nuevos frentes de colonización (Finer et al 2021; Actualidad Ambiental 2021, Praeli 2020b, 2021a). También se menciona (sin confirmación) otro grupo, menor, en algún lugar de la selva de Huánuco. Como en Colombia y en otros países, los menonitas han dado a esos asentamientos un formato empresarial, creando, en el caso de Masisea, la Asociación Colonia Menonita Cristiana Agropecuaria Masisea $^{2}$ que posee registro comercial y documentación fiscal, en la que declaran estar dedicados a la ganadería de bovinos y bufalinos.

En resumen, la actividad agropecuaria menonita en Bolivia y Paraguay ha afectado en total posiblemente más de cuatro millones de hectáreas, que han sido radicalmente desprovistas de sus bosques o de su vegeta- ción original. Estos fueron primeramente explotados, su madera fue usada en la construcción de viviendas e instalaciones o ha sido transformada en carbón o fue comercializada. No aplicaron criterios ambientales a pesar de estar ampliamente difundidos, como es proteger un porcentaje del área con su vegetación original, o preservar la vegetación ribereña. Nada cambió en sus prácticas durante o después de los años 1990, pese a los llamados de la Conferencia de las Naciones Unidas sobre Ambiente y Desarrollo de junio de 1992 (Eco-92) ni a la multitud de eventos, convenciones y tantos otros hechos, incluyendo políticas y legislaciones nacionales, llamando la atención para un uso más sensato de los recursos naturales renovables.

Ya en el siglo XXI, como visto, los menonitas han iniciado sus avances sobre la Amazonía, tanto en Bolivia como en Perú y Colombia. En total, en esos tres países ya han ocupado cerca de 40 mil hectáreas, de las que con certeza han deforestado casi 10000 apenas en Bolivia y Perú y otras tantas en Colombia. Han replicado en esos emprendimientos sus prácticas previas; es decir, han hecho tala rasa, sin dejar absolutamente ninguna vegetación natural.

\section{Otros impactos ambientales e impactos so- ciales}

Además de la deforestación y de sus bien conocidas consecuencias, en términos de pérdida de diversidad biológica, emisión de gases de efecto invernadero y alteración del ciclo hidrológico, hay otros problemas ambientales asociados a los asentamientos agropecuarios menonitas. En efecto, contrariamente a lo que

\footnotetext{
2 https://www.dnb.com/business-directory/company-profiles.asociacion_colonia_menonita_cristiana_agropecuaria_masisea.6fc7be7be7eb9cf27d1f391559b39a34.html 1
} 
puede parecer y a lo que se suele creer, salvo excepciones, no practican agricultura ecológica ni, mucho menos, producen alimentos orgánicos. En realidad, hacen un uso muy intensivo de agroquímicos de todo tipo, incluidos abonos minerales y, obviamente, pesticidas, herbicidas, fungicidas, nematicidas y otros agrotóxicos de uso común en la agricultura intensiva y, asimismo, usan semillas transgénicas siempre que pueden. Por esos motivos han sido frecuentemente denunciados. Por ejemplo, en Bolivia, se les acusó de provocar incendios forestales asociados a la limpieza de sus campos (Sputnik 2019) y, asimismo, de usar y vender maíz transgénico además de toda clase de agrotóxicos (Leisa, 2020). De México se reporta que colonias menonitas practicaron el drenaje de humedales protegidos por ley, así como sobreexplotación de acuíferos, con los consecuentes impactos para otros usuarios (Morimoto 2019). El mismo autor cita el uso abusivo de agrotóxicos en el cultivo de arroz. Según esa referencia, en 2016 un estudio del Centro de Ecología Pesquerías y Oceanografía del Golfo de México de la Universidad Autónoma de Campeche reveló la existencia de glifosato en el manto freático y en la orina de los habitantes del municipio de Hopelchen, debido al uso indiscriminado de agroquímicos. El estudio indicó que entre 1987 y 2007 se utilizaron en la región casi 2 mil toneladas de herbicida, pero que tan solo en 2016 se detectaron 13500 toneladas de ese $\mathrm{y}$ otros agroquímicos peligrosos. También relata que los residuos químicos que quedan en el aire han causado la muerte de abejas y la baja en la producción de miel en un $70 \%$.

Otro problema asociado a las colonias menonitas es el uso de maquinaria agrícola pesada, que compacta el suelo (Quadri 2017), lo que compensan con arados subsoladores, desdeñando técnicas más apropiadas para los suelos que usan, como es alternancia de cultivos o la siembra directa contribuyendo a la degradación del suelo y, por ende, a uso cada vez más intensivo de correctores, especialmente fertilizantes. Ellos no adaptan sus cultivos a la realidad ecológica local. Ganan dinero forzando la producción en base a mucha inversión convencional, especialmente en fertilizantes, pero, en el largo plazo, dejan tierra arrasada. No practican agrosilvicultura o silvopecuaria, es decir, asociaciones de árboles con cultivo o ganado, como se recomienda para los ecosistemas en que trabajan.

De otra parte, los asentamientos menonitas nuevos construyen y mantienen carreteras para acceder a sus predios. Eso facilita el ingreso de nuevos agricultores, en su mayoría invasores, que se instalan en las proximidades, ampliando el daño. Además, en el caso de Masisea los menonitas usan esa excusa para justificar las deforestaciones más recientes que achacan a terceros (Praeli 2020). Y, de ese modo también están facilitando el acceso al Área de Conservación Regional Imiría y a las tierras de una comunidad nativa de la etnia shipibo (Zero Deforestation 2020, Praeli 2021a).

La aproximación de los menonitas a los pueblos originarios tiene dos vertientes, que a veces usan simultáneamente: (i) asimilarlos a sus creencias y usarlos como mano de obra barata, como hicieron en algunos lugares del Chaco paraguayo o, (ii) empujarlos "monte adentro", sin mayor violencia, pero aprovechando el hecho de que muchos nativos no aprecian esa vecindad. Eso está ocurriendo en Masisea, Perú, con los shipibos de la comunidad aledaña de las que los menonitas invadieron una parte (Zero Deforestation 2020; Praeli 2021a) y en Puerto Gaitán, 
Meta, en Colombia (Rutas del Conflicto 2021).

Los agricultores tampoco están satisfechos con la cercanía de los menonitas. Por ejemplo, en Bolivia, el Consejo Nacional por el Cambio (Conalcam), que agrupa a sindicatos, organizaciones vecinales y otros grupos oficialistas, ha protestado reiteradamente por comportamientos atribuidos a los menonitas, especialmente uso del fuego, en el Beni y en otros lugares del país (Sputnik 2019).

\section{La ilegalidad como rutina menonita en sus prácticas agropecuarias}

Praeli (2020a, 2020b) y especialmente Praeli (2021a) han descrito bien este aspecto de la intervención menonita en la Amazonía peruana. La ilegalidad del comportamiento cubriría varios aspectos: (i) compra irregular de tierra, (ii) falta de autorización previa de cambio de uso de la tierra, (iii) deforestación sin autorización y (iv) persistencia del comportamiento ilegal a pesar de advertencias. En efecto, la primera cuestión es cómo y a quién compraron una tierra cubierta de bosques naturales que, a todas luces, es pública y que además cubren tierras de aptitud forestal, que por ley no pueden sufrir cambio de uso. A eso se suma el hecho de que pagaron precios muy variables, pero muchas veces irrisorios por la misma, basados en el engaño a pobladores locales que, de otra parte, por lo general tampoco son sus dueños legítimos sino apenas posesionarios informales. Los menonitas usan intermediarios locales para comprar la tierra, los que suelen ser personas inescrupulosas que, para efectuar las compras y ganar sus comisiones engañan y amedrentan a los agricultores. La autora mencionada examinó las partidas registrales del asentamiento de Masisea, en los que se da cuenta que el precio pagado por cada hectárea varió de 1000 soles
(307 dólares) hasta 4000 soles (1230 dólares). Pero también hay transacciones sobre áreas más grandes, demostrando la intervención ilegal de autoridades locales. En el lugar citado se detectaron 40 fichas catastrales elaboradas en el 2015 que contenían información falsa para titular bosques como si fueran áreas de cultivo. Asimismo, también en Masisea se encontró que de 47 fichas en el Sistema Catastral para Predios Rurales del Ministerio de Desarrollo Agrario y Riego (SICAR), que es la base de datos oficial que muestra información de los predios rurales de Perú, 29 de se superponen con los territorios de dos comunidades del pueblo shipibo-konibo; cuatro con los de la comunidad nativa Caimito y 25 con las tierras de la comunidad nativa Buenos Aires.

La siguiente irregularidad es el cambio de uso de la tierra y la deforestación sin pedir permiso, sin haber recibido la autorización pertinente o, eventualmente, antes de recibirla. Estas irregularidades estarían confirmadas por las autoridades forestales locales y por la Procuraduría del Medio Ambiente y las Fiscalías Especializadas en Materia Ambiental (FEMA) de Ucayali y Loreto que investigan la pérdida de bosque en los dos territorios (Praeli 2020b, 2021a). Por ejemplo, en el informe técnico sobre Tierra Blanca, al que también accedió la autora, se precisa que en esa zona deforestada existen 135 predios privados y solo 12 permisos forestales, no todos de menonitas. La ley dispone que primero se acepta el cambio de uso de la tierra y que, estando eso resuelto, debe pedirse la autorización de desbosque. Son dos pasos que han sido ignorados. Finalmente, los menonitas fueron advertidos y alertados sobre los pasos legales a cumplir, pero ignoraron y han continuado ignorándolos. En todos los casos ha sido evidente la participación de autoridades locales 
corruptas, gubernamentales o municipales.

La rutina parece ser, para los menonitas, no complicarse la vida solicitando autorizaciones para cambiar de uso de la tierra y derrumbar el bosque, tal como lo revela su comportamiento también en México, donde tienen un largo historial de violaciones de la ley. No hay información de cuántas hectáreas ellos tienen, pero deben poseer un área muy grande. En décadas recientes han expandido sus actividades y se han ido desplazando a estados como Baja California, Oaxaca, Tabasco y Yucatán. Estas expansiones han generado muchos conflictos ambientales debido a deforestación de bosques naturales, algunos de ellos protegidos, sin ninguna autorización. Quadri (2017) cita varios casos, entre 2008 y 2017, como la deforestación de 800 hectáreas de bosque tropical en Campeche y, otra vez, en 2013, cuando la Procuraduría General del Ambiente (Profepa) advirtió la destrucción sin ningún permiso de 2300 hectáreas de bosque en predios menonitas del estado Cohauila. Entre 2012 y 2013, en el estado de Quintana Roo, la Profepa inspeccionó y denunció penalmente a un grupo de menonitas por un desmonte con fuego (incendio provocado en el bosque tropical) y cambio de uso de suelo sin autorización que afectó casi 100 hectáreas dentro de un área natural protegida (Quadri 2017). El mismo autor indica que, en 2017, también en el estado de Quintana Roo, la Profepa conjuntamente con la Secretaría de la Marina Armada de México, constató la destrucción de 1445 hectáreas de bosque tropical en tres predios de menonitas, de manera ilegal y sin ningún tipo de autorización. A 2017, la Profepa había levantado más de 176 expedientes en contra de las comunidades menonitas de México, por afectaciones ecológicas. Otro autor (Morimoto 2019) señala que, en 2017, la
Secretaría del Medio Ambiente de Campeche registró la deforestación de 759 hectáreas de la selva baja y la consecuente clausura de 5 aserraderos y el decomiso de 299 hornos para carbón vegetal. Asimismo, ese autor señala la sobreexplotación de acuíferos para cultivar arroz y abuso de aplicación de agrotóxicos. La Asociación de Consumidores Orgánicos de México los ha denunciado reiteradamente (Consumidores Orgánicos 2019). También se ha responsabilizado a los menonitas por la invasión y destrucción de la vegetación de 1750 hectáreas de un humedal de Quintana Roo (Fitzmaurice 2017) acción que, aparentemente continuó, alcanzando más de 3 mil hectáreas en 2019. En Bacalar, Quintana Roo, se les sancionó por haber removido la vegetación de 679 hectáreas, afectando un ecosistema de selva mediana subperennifolia y selva espinosa subperennifolia de Astornium graveolens, que era refugio de Thrinax radiata y Aratinga nana, especies listadas en la Norma Oficial Mexicana NOM-059-SEMARNAT-2010, sobre especies raras o amenazadas (Cambio 22 2020). Las mismas acusaciones de violar la legislación, no solo en relación a las tierras y a la deforestación, sino asimismo por contaminación ambiental, abuso del uso de agua en detrimento de terceros han sido reportadas en Bolivia (Leisa 2020). Traspasso (1994) concluye que la inmigración menonita en Belice ha sido una fuerza destructiva mayor.

Todas las referencias a la ilegalidad del proceder menonita abundan en denunciar la aparente inmunidad con la que actúan, a pesar de la reiteración de las denuncias y de las sanciones, que no respetan. Parecen acudir a argumentos e influencias religiosas y, en el caso de México, se beneficiarían de las mismas reglas que privilegian las etnias nativas (Quadri 2017). 


\section{CONCLUSIONES}

La primera conclusión de esta revisión es que la idea tan generalizada de que los menonitas son una secta apegada a la naturaleza, que practica una agricultura "ecológica" u "orgánica" es falsa. Esta visión proviene de uno de los subgrupos menonitas, conocidos como amish, instalados principalmente en Pensilvania, EE.UU., pero que en realidad viven también en muchos otros lugares, inclusive en América Latina. Estos, en efecto, viven muy simplemente y ejercen una agricultura rudimentaria, de bajo impacto. Pero los demás, aunque llevan una vida sin extravagancias, practican una agricultura moderna, intensiva, de alto impacto ambiental, con uso y abuso de agroquímicos, maquinaria agrícola pesada y técnicas agronómicas de alto impacto, incluido uso generalizado de variedades de cultivos genéticamente modificados.

Su impacto ambiental más importante ha sido como agentes eficientes de deforestación y alteración de ecosistemas naturales. En base a las informaciones previas se puede deducir que a nivel de América del Sur han deforestado directamente no menos de cuatro millones de hectáreas, principalmente en los biomas Chaco (Paraguay y Bolivia) y Chiquitanía (Bolivia) y que continúan haciéndolo. Como suelen instalarse en tierras nuevas, construyen vías de acceso y éstas son aprovechadas por otros agricultores informales que aumentan la extensión de la deforestación. Preocupa, especialmente, la expansión de sus programas de colonización en las regiones amazónicas de Bolivia, Perú y Colombia, donde ya eliminaron probablemente unas veinte mil hectáreas de bosques, lo que continúan haciendo sin interrupción. Sorprende el hecho de que en sus operaciones no aplican ningu- na salvaguarda ambiental, como establecimiento de reservas de bosque o la protección de la vegetación de riberas o de las nacientes de agua. Parecen ignorar completamente la importancia de desarrollar una agricultura más balanceada con el entorno natural.

Preocupa, asimismo, su comportamiento social. Ellos violan sistemáticamente $y$, en general, impunemente las leyes de ocupación y uso de la tierra que rigen en los países a los que llegan, pareciendo reclamar privilegios especiales que los exoneran de obedecerlas. En el caso de la Amazonía del Perú, la acción de los menonitas se suma a la de empresas transnacionales que, con el pretexto de desarrollar plantaciones de cacao y palma aceitera, han usado las mismas prácticas ilegales para ocupar la tierra en desmedro de los pobladores locales, especialmente los indígenas. De hecho, las colonias menonitas han perjudicado directamente a comunidades nativas de todos los países a los que llegaron.

De otro lado, es innegable que las inmigraciones menonitas han brindado lo que puede ser considerado beneficio a algunos países. Así, ellos convirtieron al Paraguay en una potencia mundial exportadora de carne de bovinos y, en el caso de Bolivia, han contribuido significativamente a transformarlo en país exportador de soya y otras commodities. No hay duda que, en esos dos países, los menonitas han tenido una gran influencia en la economía $y$, por ende, en la política. Pero, eso, como visto, ha tenido un alto costo ambiental y social.

En el caso especial del Perú hay, pues, preguntas obvias que merecen ser respondidas por las autoridades pertinentes, tanto nacionales como regionales: (i) ¿cómo los menonitas compraron tierra con bosques naturales, ha- 
bida cuenta que, en principio, esos bosques son del Estado o de comunidades nativas?, (ii) ¿cómo esas compras fueron formalizadas, registradas o legalizadas si, en realidad son ilegales?, (iii) ¿por qué las autoridades competentes esperaron a que se deforesten, en la actualidad unas cuatro mil hectáreas, sin hacer nada o casi nada?, y (iv) ¿qué autoridades o influencias han facilitado la entrada masiva al Perú de los menonitas y su asentamiento en los departamentos de Loreto y Ucayali?

Finalmente, como es bien sabido, los propios peruanos son grandes devastado- res de sus bosques naturales, estando ya próximos de llegar a la destrucción del $20 \%$ de su parte de la Amazonia (Dourojeanni 2019), muy cerca del llamado punto de inflexión, a partir del cual el proceso de degradación de ese bioma puede tornarse irreversible (Lovejoy $\mathrm{y}$ Nobre 2018). Es decir que, ciertamente, no es deseable que en ese contexto se agregue la deforestación que realizan, en este caso, nuevos grupos extranjeros que, con muchos más recursos, brindan pésimo ejemplo a las poblaciones locales.

\section{REFERENCIAS BIBLIOGRÁFICAS}

Actualidad Ambiental. 2021. Noticia. Ucayali: detectan nueva deforestación de 159 hectáreas en zona de colonia menonita. SPDA, Lima. Martes 24 de Agosto https://www.actualidadambiental.pe/investigacion-a-menonitas-por-nuevas-159-hectareas-deforestadas/

Betancourt O. 2018. Los menonitas buscan casa en Colombia. Tierras de América. http://www.tierrasdeamerica.com/2018/04/14/los-menonitas-buscan-casa-en-colombia-adquieren-grandes-extensiones-de-tierra-en-meta-una-region-donde-hubo-graves-conflictos-entre-la-guerrilla-y-los-paramilitares-venimos-aqui-para-queda/

Cambio 22. 2020. Tras años de denuncias por ecocidio, PROFEPA multa a la Comunidad Menonita de Bacalar. https://cambio22.mx/tras-anos-de-denuncias-por-ecocidio-profepa-multa-a-la-comunidad-menonita-de-bacalar/

Cassel, DK. 2016. History of the Mennonites: Historically and biographically arranged from the time of the reformation; More particularly from the time of their emigration to America. Classic Reprint Series. Forgoten Books. 478p.

Consumidores Orgánicos. 2019. https://consumidoresorganicos.org/2019/06/13/la-comunidad-menonita-extiende-su-frontera-agricola-echando-la-selva-abajo/

Correia JE. 2020. Mennonites helped turn Paraguay into a mega beef producer - indigenous people may pay the price The Conversation March 10, 2020. https://www.ft.com/content/c4283204-927d-11e9-aea1-2bld33ac3271 
De Syl, V.; Herold M; Achar, F; Beuchle R; Clevers JGPW; Lindquist E. and Verchot, L. 2015. Land use patterns and related carbon losses following deforestation in South America. Environ. Res. Lett. 10124004. https://iopscience.iop.org/article/10.1088/1748-9326/10/12/124004/pdf

Dorst, J. 1965. Avant que Nature Meure, pour que Nature Vive. Delachaux, Paris. 544p.

Dourojeanni, MJ. 2019a. peruana ¿Qué futuro? (en línea). Lima, Perú, Grijley. https://www.academia.edu/39962447/Amazonia_Qu\%C3\%A9_Futuro

Dourojeanni, MJ. 2020. Los menonitas y el ambiente en América Latina. Actualidad Ambiental. SPDA. Lima. https://www.actualidadambiental.pe/opinion-menonitas-y-el-ambiente-en-america-del-sur/

Duerksen H. 2011. Los menonitas, protagonistas de la historia del Chaco y Paraguay. ABC Color, Asunción.

Dyck, CJ. 1993. Introduction to Mennonite History. Herald Press. 456p.

Fast, Kerry. 2020. "Review of: Giesbrecht, Kennert. 2018. Strangers and Pilgrims: How Mennonites Are Changing Landscapes in Latin America. Vol. 2. Trans. James Schellenberg. Steinbach, MB: Die Mennonitische Post. 303pp., h/c, \$29.95 CAN." Journal of Amish and Plain Anabaptist Studies 8(1):99-100.

https://ideaexchange.uakron.edu/amishstudies/vol8/iss1/11/

Finer M y N Mamani. 2019. Colonias Menonitas: Nuevo driver de deforestación en la . MAAP: 112. Proyecto de Monitoreo de los Andes Amazónicos. https://maaproject.org

Finer M., Mamani N. y Suarez D. 2020. MAAP: Colonias Menonitas continúan la gran deforestación en la Amazonia Peruana. Proyecto de Monitoreo de los Andes Amazónicos. https://maaproject.org.

Fitzmaurice, A. 2017. Atribuyen a comunidad menonita daño de 1,450 hectáreas a la zona de Bacalar. https://www.puntomedio.mx/atribuyen-a-comunidad-menonita-dano-1450-hectareas-a-la-zona-bacalar/

Gallirgos R. 2015. El racismo peruano. In El racismo en el Perú. Ed. J C Agüero. Serie diversidad cultural 9. Ministerio de Cultura. P.83-152 https://centroderecursos.cultura.pe/sites/default/files/rb/pdf/el-racismo-peruano.pdf 
GAMEO. 2015. Global Anabaptist Mennonite Encyclopedia on line: World Mennonite Membership Distribution.

https://gameo.org/index.php?title=World_Mennonite_Membership_Distribution

Giesbrecht, K. 2018. Strangers and Pilgrims Volume II: How Mennonites are changing landscapes in Latin America. Die Mennonitische Post.

Giesbrecht, K., \& Klassen, W. 2015. Auf den Spuren der

Mennoniten: $19.000 \mathrm{~km}$ durch Amerika. Die Mennonitische Post. https://www.biblio.com/book/spuren-mennoniten-giesbrecht-kennert-wilfried-klassen/

Goodland RJA; Irwin, HS. 1975. Amazon Jungle: Green Hell to Red Desert? Elsevier Scientific, NY. 155p.

Goossen BW. 2016. Religious nationalism in an age of globalization: The Case of Paraguay's "Mennonite State". Almanack no.14 Guarulhos Sept./Dec. 2016. On-line version ISSN 2236-4633. https://doi.org/10.1590/2236-463320161405

Hecht A. 1976. The agricultural economy of the Mennonite settlers in Paraguay: Impact of a road. Ekistics, 42(248), 42-48. http://www.jstor.org/stable/43618730.

Kopp A. 2015 Las colonias menonitas en Bolivia. Tierra, LaPaz. http://www.ftierra.org/index.php/publicacion/libro/147-las-colonias-menonitas-en-bolivia.

La Liga Contra el Silencio, Mongabay Latam, Rutas del Conflicto. 2021. Colombia: menonitas deforestan un territorio ancestral en el Meta. Mongabay. 2021. 31 mayo 2021 https://es.mongabay.com/2021/05/colombia-menonitas-deforestan-un-territorio-ancestral-en-el-meta/

Leisa. 2020. Bolivia: Menonitas venden maíz transgénico subvencionado. Leisa, La Paz http:// www.leisa-al.org/web/index.php/lasnoticias/biodiversidad/804-bolivia-menonitas-venden-maiz-transgenico-subvencionado.

IMPACTO. 2020. Responsabilizan a colonia religiosa menonita de talar 7.500 hectáreas de selva en la Amazonia. Semana Sostenible, Impacto IAB. Domingo, 8 de noviembre de 2020. Bogotá. https://sostenibilidad.semana.com/impacto/articulo/tres-colonias-del-grupo-religioso-menomita-acabaron-con-7500-hectareas-de-selva-en-peru-y-bolivia/47470 
le Polain de Waroux, Y., Neumann J., O’Driscoll A., and Schreiber, K. 2020. Pious Pioneers: The Expansion of Mennonite Colonies in Latin America. Journal of Land Use Science, December 15, 2020, 1-17. https://doi.org/10.1080/1747423X.2020.1855266.

Lovejoy TE, \& Nobre C. (2018). Amazon tipping point. Science Advances, 4(2). https://advances.sciencemag.org/content/4/2/eaat2340.full

Luz A G, I A Gomes, C L Löwen Sahr. 2014. Os Padrões de povoamento Menonita no Brasil: Análise da Colônia Witmarsum. Anais Semana de Geografia. Vol. 1, Num. 1. Ponta Grossa: UEPG, 2014. ISSN 2317-9759.

Maguiña, E. 2010. Un acercamiento al estudio de las inmigraciones extranjeras en el Perú durante el siglo XIX y las primeras décadas del siglo XX. Revista "Tierra Nuestra" UNALM: 8(1):65-96. DOI: http://dx.doi.org/10.21704/rtn.v8i1.100. https://revistas.lamolina.edu.pe/index.php/tnu/article/view/100

Mander B. 2019. Paraguay's Mennonites struggle with an influx of new neighbors. Financial Times https://www.ft.com/content/c4283204-927d-11e9-aea1-2bld33ac3271

Moya C. 2020. Reseña sobre los menonitas en Ecuador. Iglesia Cristiana Anabautista Menonita De Ecuador. http://anabautistasenecuador.weebly.com/historia-menonitas-ecuador. html

Praeli YS. 2020a. Menonitas en Perú: fiscalía sorprende a grupo talando sin autorización y ordena paralizar deforestación en Loreto. Mongabay,18 noviembre 2020. https://es.mongabay.com/2020/11/menonitas-peru-deforestacion-loreto/

Praeli YS. 2020b. Menonitas en Perú: fiscalías de Loreto y Ucayali investigan deforestación de 2500 hectáreas en la . Mongabay Latam. 27 octubre 2020. https:/es.mongabay.com/2020/10/menonitas-peru-investigacion-deforestacion-amazonia/

Praeli YS. 2021a. Menonitas en Perú: la historia oculta de la entrega de bosques en Masisea. Mongabay, 9 abril 2021. https://es.mongabay.com/2021/04/menonitas-peru-historia-entrega-bosques-masisea/

Praeli, YS. 2021b. Perú: fiscalía confirma tala ilegal en bosque intacto tras invasión de asociación de israelitas. Mongabay, 6 mayo 2021. https:/es.mongabay.com/2021/05/peru-tala-ilegal-invasion-israelitas/

Ritchie H., Roser., M. 2021. Forests and Deforestation. Published online at OurWorldInData. org. https://ourworldindata.org/forests-and-deforestation 
Rutas del Conflicto. 2021. Los menonitas acumulan en los llanos una tierra con pasado turbio. https://rutasdelconflicto.com/notas/los-menonitas-acumulan-los-llanos-tierra-pasado-turbio

Schartner, S., \& Dürksen, S. 2009. Bolivien, Zufluchtsort der konservativen Mennoniten (Bolivia, refugio de los menonitas conservadores). Santa Cruz, Bolivia: Comité Central Menonita.

Sputnik. 2019. Movimientos sociales bolivianos denuncian a menonitas y brasileños por incendios. La Paz. 3 septiembre, 2019. https://www.elpais.cr/2019/09/03/movimientos-sociales-bolivianos-denuncian-a-menonitas-y-brasilenos-por-incendios/

Trapasso L M. 1994. Indigenous attitudes, ecotourism, and Mennonites: Recent examples in rainforest destruction/preservation. GeoJournal 33 (4): 449-452. doi:10.1007/ BF00806428. https://link.springer.com/article/10.1007/BF00806428

El Tiempo. Unidad Investigativa. 2018. Unos 300 menonitas ya han desembolsado $\$ 64.000$ millones para hacerse con tres megafincas. La poderosa congregación que ha comprado 16.000 hectáreas en el Meta. El Tiempo, Bogotá. https://www.eltiempo.com/justicia/investigacion/colonia-menonita-compra-extensos-terrenos-en-meta-202530

Vidal J. 2010. Chaco deforestation by Christian sect puts Paraguayan land under threat. The Guardian. October 5, 2020. https://www.theguardian.com/world/2010/oct/05/chaco-paraguay-deforestation\#: : :text=The $\% 20$ large $\% 20$ Mennonite $\% 20$ families $\% 20$ and,businesses $\% 20$ dominating $\% 20$ Paraguayan $\% 20$ livestock $\% 20$ farming.

Villasante M. 2020. FREPAP: un partido ultra religioso colonizador nefasto de la . Breve análisis de los resultados electorales. Servindi. https://www.servindi.org/18/02/2020/frepap-un-partido-ultra-religioso-colonizador-nefasto-de-la-amazonia.

Wikipedia. 2021. Mennonites. https://en.wikipedia.org/wiki/Mennonites

Wikipedia. 2021a. Menonitas. https://es.wikipedia.org/wiki/Menonita

Wikipedia. 2021c. Menonitas en Perú. https://es.wikipedia.org/wiki/Menonitas_en_Per\%C3\%BA

Zero Deforestation. 2020. Un nuevo desastre para los indígenas del Perú: ¡los menonitas! http://www.zero-deforestation.org/p_un_nuevo_desastre_para_los_indigenas_del_ peru_los.htm 